\title{
Lysosomal storage disorders: Diagnostic dilemmas and prospects for therapy
}

David A. Wenger, PhD, Stephanie Coppola, and Shu-Ling Liu, MD

\begin{abstract}
Purpose: The main purpose of this review is to address some concerns regarding the accurate and timely diagnosis of lysosomal storage disorders (LSD). Methods: Using their experience in diagnosing LSD in more than 2500 individuals, the authors highlight several diagnostic difficulties and solutions and review the latest methods for early diagnosis and treatment. Results: While "classic" patients can be accurately diagnosed using relatively simple methods in an experienced laboratory, atypical patients require more detailed studies. With a few exceptions, almost all LSD can be diagnosed in leukocytes or plasma. Methods for screening all newborns without a family history of a LSD have been proposed, but such screening may require a large amount of effort for little gain. Conclusions: With effective therapy becoming available for some LSD, early diagnosis is critically important. If the goal is to prevent serious complications related to the nervous and skeletal systems, earlier diagnosis is potentially advantageous. Accurate prognosis and assessing the need for aggressive therapy in newly diagnosed patients are problems that need further study. Genet Med 2002:4(6):412-419.
\end{abstract}

Key Words: lysosomal storage diseases, enzyme replacement therapy, hematopoietic stem cell transplantation, phenotype prediction, prenatal testing

The lysosomal storage disorders (LSD) constitute a heterogeneous group of genetic diseases affecting humans from the fetal period to adulthood. They are caused by mutations in approximately 40 genes coding for proteins eventually involved in the lysosomal hydrolysis and transport of normal cellular compounds. While most lysosomal disorders thus far delineated result from mutations in genes coding for hydrolyzing enzymes, others are caused by mutations in genes coding for so-called activator proteins, transport proteins, and enzymes required for the posttranslational processing of lysosomal enzymes. Molecular analysis has identified many mutations resulting in decreased functionality, and this results in a wide clinical spectrum for patients with a deficiency in the same required protein. Early diagnosis is becoming more critical as effective therapy becomes a reality for some disorders. Enzyme replacement therapy (ERT) or hematopoietic stem cell transplantation (HSCT) is being performed on an everexpanding number of patients. The results, while promising for some of these disorders, are still not universally successful, and many patients are not eligible for these treatments because they are too mentally or physically impaired when diagnosed.

\footnotetext{
From the Department of Neurology, Jefferson Medical College, Philadelphia, Pennsylvania. To obtain more information about sending samples, please visit the authors' web site, www.tju.edu/lysolab.

David A. Wenger, PhD, Department of Neurology, Jefferson Medical College, 1020 Locust Street, Room 394, Philadelphia, PA 19107.

Received: July 8, 2002.

Accepted: August 15, 2002.
}

DOI: 10.1097/01.GIM.0000037284.24656.5E
Improvements in diagnostic methods as well as heightened physician awareness may significantly increase the number of patients undergoing some form of corrective therapy. In addition, a diagnosis provides opportunities for genetic counseling and prenatal testing in future pregnancies.

The incidence of LSD is estimated to be approximately 1 in every 5000 to 8000 births in the United States, Europe, and Australia. ${ }^{1-3}$ In the United States this would mean that 500 to 800 individuals with the potential for developing a LSD are born each year. The presence of signs and symptoms recognized by a physician followed by a confirmatory diagnosis in a qualified laboratory constitutes the most irrefutable definition of disease. The course of action would depend on the stage of the disease and the availability of treatment. While it is generally accepted that treatment should begin before serious physical and/or mental deterioration is present, some individuals with very mild disease might not be candidates for expensive or potentially dangerous therapy.

In this article we will review the current strategies for diagnosing LSD and the options currently available for therapy. It should be noted that this laboratory does not test for all LSD, and this article will focus mainly on those disorders in which we have considerable diagnostic experience. There are several excellent reviews that describe the wide range of clinical features of patients with LSD and detail the biochemistry of the pathways involved in the lysosomal catabolism of complex carbohydrates, lipids, and glycosaminoglycans. ${ }^{4-6}$ Individual chapters on these disorders in the latest version of The Metabolic and Molecular Bases of Inherited Disease are very informative. 


\section{Key clinical features that should signal a request for a screen for LSD}

Patients with one of the LSD can range from neonates to adults in the ninth decade of life. The symptoms can include obvious signs for a storage process to very mild mental or visceral changes. Children born with nonimmune fetal hydrops (NIFH) or significant hepatosplenomegaly (HSM) are candidates for a screen for LSD. ${ }^{7}$ We arrive at a definitive diagnosis in approximately $10 \%$ of samples received from pregnancies that result in NIFH. The lysosomal causes are mucopolysaccharidosis VII (MPS VII; $\beta$-glucuronidase deficiency), GM1 gangliosidosis, mucolipidosis II (I-cell disease), Niemann-Pick Type C, Gaucher disease, sialic acid storage disease, sialidosis, and galactosialidosis. The diagnosis of these patients can be made using cultured cells obtained from samples taken in utero or from blood and/or cultured cells obtained after birth. While there is little help to be provided to the affected baby, the value of a definitive diagnosis for genetic counseling purposes is worth the effort. This will allow for risk prediction in future pregnancies, early prenatal diagnosis, and information for family members. The presence of jaundice at birth is seen in a large number of patients with classic Niemann-Pick Type C. ${ }^{8}$ They usually respond to phototherapy and may have no other clinical features until the middle of the first decade or later.

Most samples come from patients under 5 years of age; however, an increasing number of samples are sent from older individuals, including adults. The symptoms and clinical features that would suggest a screen for LSD are lost developmental milestones after a period of relative normalcy, progressive weakness, ataxia, vision loss, development of HSM, coarse facial features, evidence of bone disease such as dysostosis multiplex, and onset of eye changes such as corneal clouding or macular cherry red spots. Other subtle clinical features found in some patients are used by this laboratory to help with test selection. ${ }^{9}$ From the approximately 30,000 patient samples received in this laboratory over the past 29 years, we have reached a definitive diagnosis in more than 2500 cases, a "hit" rate of approximately $8 \%$.

\section{Obtaining a diagnosis in a patient}

When a physician suspects a patient may have a LSD, samples must be sent to a laboratory performing such testing. The LSD tested for in this laboratory are noted in Table 1. In this laboratory most of the testing is done using leukocytes and/or plasma isolated from a whole blood sample. ${ }^{9}$ While much of the same testing is available using cultured skin fibroblasts, this adds both time and expense to the process. Only a few LSD, including Niemann-Pick Type C, sialidosis, and Farber disease, require fibroblasts for a definitive diagnosis. Most testing is based on the measurement of the activities of a panel of enzymes in leukocytes determined by the clinical information supplied. As there is significant overlap in clinical features between different diseases and significant variability within a given disorder, help with test selection is provided by this laboratory because of our considerable experience diagnosing typical and atypical patients. If one test shows very low activity while other values are normal, the diagnosis is usually complete. Some important exceptions will be discussed below.

A role for studies in urine as a prelude to enzyme analysis in the diagnosis of LSD may be of value. If a child has coarse facial features and dysostosis multiplex, a screen of enzymes involved in the lysosomal degradation of glycosaminoglycans (GAGS) and oligosaccharides (OLS) is indicated. A positive urine screen for GAGS and OLS may be useful, but enzyme testing to determine the specific diagnosis is still required. Also, the incidence of false-negative and false-positive results remains a problem. A definitive diagnosis has not been made in a significant number of patients who had "abnormal OLS bands in urine" or a weak positive test for GAGS. On the other hand, a negative urine screen for these analytes does not necessarily rule out a diagnosis of one of these disorders.

When does a low value for one enzyme in a leukocyte enzyme screen not result in a definitive diagnosis? This is true for only a few disorders. One of these is galactosialidosis or combined $\beta$-galactosidase/sialidase deficiency. ${ }^{10,11}$ Because sialidase activity cannot be reliably measured using previously frozen leukocytes, only $\beta$-galactosidase activity is initially measured in an enzyme screen. If it is less than $10 \%$ of our normal mean, a diagnosis of either GM1 gangliosidosis or galactosialidosis should be considered. However, only in GM1 gangliosidosis would plasma $\beta$-galactosidase activity also be low. Since parents of all diagnosed patients should have their enzyme levels measured, it will be noted that the parents of patients with galactosialidosis do not have carrier levels of $\beta$-galactosidase activity. Both $\beta$-galactosidase and sialidase activities will be severely deficient when assayed in previously unfrozen cultured skin fibroblasts.

Metachromatic leukodystrophy (MLD) is another disease in which a low leukocyte or fibroblast enzyme value is not necessarily the diagnosis. About one in seven individuals carry the so-called pseudodeficiency (Pd) allele for arylsulfatase A. Therefore, about 1 in 200 people will have low (5-15\% of our normal mean) arylsulfatase A activity. ${ }^{12-14}$ Unfortunately, the finding of the Pd mutation by molecular analysis does not rule out MLD since a significant number of families have a MLDcausing mutation on the same allele as the Pd mutation. ${ }^{15}$ Such a family is presented in Figure 1. In individuals with low arylsulfatase A activity, the finding of excessive amounts of sulfatides in urine usually confirms the diagnosis of MLD. Pseudodeficient individuals do not excrete excess sulfatides. Since about 1 in 14 parents of patients with MLD have quite low arylsulfatase A activity (having one MLD-causing allele and one Pd allele), it is critical that parental arylsulfatase A values be obtained before prenatal diagnosis is attempted and before other offspring, especially those younger than the proband, are tested. This could help to eliminate a potential problem with interpretation, as the low arylsulfatase A values measured in individuals who are genotypically MLD/MLD, $\mathrm{Pd} / \mathrm{Pd}$, and MLD/Pd overlap. In addition, it should be noted that patients with multiple sulfatase deficiency also have low arylsulfatase A activity and excrete sulfatides plus GAGS in urine. However, 
Table 1

Lysosomal storage diseases and the current treatment options

\begin{tabular}{|c|c|c|}
\hline Name of disease & Defective protein & Treatment options $^{a}$ \\
\hline \multirow[t]{2}{*}{ GM1 gangliosidosis, MPS IVB ${ }^{b}$} & \multirow[t]{2}{*}{ Acid $\beta$-galactosidase } & SC, ${ }^{c} \mathrm{HSCT}, \mathrm{ERT}$ for MPS \\
\hline & & IVB \\
\hline GM2 gangliosidosis, Tay-Sachs ${ }^{b}$ & Hexosaminidase A & SC, HSCT \\
\hline GM2 gangliosidosis, Sandhoff ${ }^{b}$ & Hexosaminidase A \& B & SC \\
\hline GM2 gangliosidosis, $\mathrm{AB}$ variant ${ }^{b}$ & GM2 activator protein & SC \\
\hline Fabry disease ${ }^{b}$ & $\alpha$-Galactosidase & SC, ERT, HSCT \\
\hline Gaucher disease $^{b}$ & Glucocerebrosidase & SC, ERT, HSCT \\
\hline Niemann-Pick Types A \& $\mathrm{B}^{b}$ & Sphingomyelinase & SC, HSCT and ERT for Type B \\
\hline Niemann-Pick Type $\mathrm{C}^{b}$ & NPC1 & SC, HSCT \\
\hline Niemann-Pick Type C2 ${ }^{b}$ & NPC2 & SC \\
\hline Metachromatic leukodystrophy ${ }^{b}$ & Arylsulfatase A & SC, HSCT \\
\hline Krabbe disease $^{b}$ & Galactocerebrosidase & SC, HSCT \\
\hline$\alpha$-Mannosidosis ${ }^{b}$ & $\alpha$-Mannosidase & SC, HSCT \\
\hline$\beta$-Mannosidosis ${ }^{b}$ & $\beta$-Mannosidase & SC \\
\hline Sialidosis, mucolipidosis $\mathrm{I}^{b}$ & Sialidase & SC \\
\hline Sialic acid storage disease, Salla ${ }^{b}$ & Transport protein & SC \\
\hline Galactosialidosis $^{b}$ & Protective protein & SC \\
\hline Fucosidosis $^{b}$ & $\alpha$-L-Fucosidase & SC, HSCT \\
\hline MPS I (Hurler, Scheie) ${ }^{b}$ & $\alpha$-L-Iduronidase & SC, HSCT, ERT \\
\hline MPS II (Hunter) & Iduronate-2-sulfatase & SC, HSCT, ERT \\
\hline MPS IIIA (Sanfilippo) & Glucosamine- $N$-sulfatase & SC, HSCT \\
\hline MPS IIIB ${ }^{b}$ & $\alpha$-N-Ac-glucosaminidase & SC \\
\hline MPS IIIC & AcCoA: $\alpha$-glucosaminide- $N$-acetyltransferase & SC \\
\hline MPS IIID & $\mathrm{N}$-acetylglucosamine-6-sulfatase & SC \\
\hline MPS IVA (Morquio A) & $\mathrm{N}$-acetylgalactosamine-6-sulfatase & SC \\
\hline MPS VI (Maroteaux-Lamy) ${ }^{b}$ & Arylsulfatase B & SC, HSCT, ERT \\
\hline MPS VII ${ }^{b}$ & $\beta$-Glucuronidase & SC, ERT \\
\hline Mucolipidosis II \& III ${ }^{b}$ & UDP-N-Ac-glucosaminyl phosphotransferase & SC, HSCT \\
\hline Mucolipidosis IV & Mucolipin 1 & SC \\
\hline Multiple sulfatase deficiency ${ }^{b}$ & Sulfatase modifier protein & SC \\
\hline Pyknodysostosis & Cathepsin K & SC \\
\hline Cystinosis & Cystine transport protein & SC, cysteamine \\
\hline
\end{tabular}

-Continued

the parents of these patients do not have carrier levels of these other sulfatases. Correct disease assignment and accurate prenatal testing are not difficult once the studies described above have been carried out.

It should be noted that some rare patients with clinical features resembling juvenile MLD have a defect in a so-called sphingolipid activator protein known as saposin B (SAP1). ${ }^{16,17}$ This sphingolipid activator protein facilitates the interaction between the lipid substrate sulfatide and arylsulfatase A.
These patients have normal arylsulfatase A activity measured with synthetic substrate and radiolabeled sulfatide (with detergents present). To make a presumptive diagnosis, urine should be examined for sulfatide. If sulfatide is being excreted and arylsulfatase A is normal, a defect in saposin B can be suspected. DNA analysis of the saposin $B$ region of the prosaposin gene could possibly identify the mutation(s).

Some other LSD require more than routine leukocyte enzyme studies to arrive at a definitive diagnosis. These include 
Table 1

Continued

\begin{tabular}{|c|c|c|}
\hline Name of disease & Defective protein & Treatment options ${ }^{a}$ \\
\hline Neuronal ceroid lipofuscinosis 1 & Palmitoyl-protein thioesterase 1 & SC, HSCT \\
\hline Neuronal ceroid lipofuscinosis 2 & Tripeptidyl peptidase 1 & SC \\
\hline Saposin defect, MLD type ${ }^{b}$ & Saposin B & SC \\
\hline Saposin defect, Gaucher type ${ }^{b}$ & Saposin C & SC \\
\hline Saposin defect, generalized type ${ }^{b}$ & Prosaposin & SC \\
\hline Aspartylglucosaminura & Aspartylglucosaminidase & SC \\
\hline Farber disease $^{b}$ & Acid ceramidase & SC, HSCT \\
\hline Wolman disease, cholesterol ester storage disease (CESD $)^{b}$ & Acid lipase & SC, HSCT, liver transplant for CESD \\
\hline Schindler disease & $\alpha$-N-acetylgalactosaminidase & SC \\
\hline Pompe disease & $\alpha$-Glucosidase & SC, ERT \\
\hline
\end{tabular}

${ }^{a}$ HSCT is not available for all patients with a given diagnosis, and ERT may be in use or only at the stage of preclinical trials. The use of these treatments in a few patients does not necessarily indicate a successful outcome.

${ }^{b}$ Diseases diagnosed in the authors' laboratory.

${ }^{c}$ Supportive care indicates any procedure performed to alleviate pain, discomfort, and seizures and may include splenectomy and kidney transplantation when indicated.

Niemann-Pick Type C (NPC) as well as LSD caused by activator proteins and transport proteins. Patients with NPC are clinically heterogeneous, ranging from neonates with NIFH to adults with dystonia and mild clinical features. ${ }^{8}$ There are more patients with NPC than patients with Niemann-Pick Types A and B caused by sphingomyelinase deficiency. Defects in two genes, NPC1 and NPC2, have been demonstrated to be responsible for NPC. ${ }^{18,19}$ The definitive diagnosis of NPC requires studies in cultured skin fibroblasts, except in those genetic isolates in which only one mutation is responsible for almost all of the cases (see below). The best screening test involves staining cultured cells with filipin to detect free cholesterol. ${ }^{20}$ Cells from "classic" patients have intense perinuclear staining, and cholesterol esterification studies reveal a severe reduction in the ability to esterify cholesterol at all time points. These cells usually have a partial reduction in sphingomyelinase activity (25-60\% of normal mean). Accurately diagnosing the "variant" patients presents a greater problem. Filipin staining may be only weakly positive, and cholesterol esterification can be partially deficient in short time studies (5-6 hours) but near normal after 24 hours. In addition, sphingomyelinase activity can be within the normal range. Molecular analysis of the NPC1 gene has identified a large number of mutations, with only one occurring in a significant number of patients with European heritage. ${ }^{21}$ This same mutation (I1061T) is responsible for almost all of the Hispanic patients whose ancestors come from the Rio Grande of Northern New Mexico and Southern Colorado. All prenatal, postnatal, and carrier testing in this population is done by polymerase chain reaction-based mutational analysis.

A small number of patients have defects in so-called sphingolipid activator proteins. Saposin B-deficient MLD has been discussed above. Saposin B is one component of prosaposin, a protein coded for by a gene on chromosome 10 that is pro- cessed into four separate saposins labeled A-D. ${ }^{22}$ These four relatively small proteins, consisting of about 80 amino acids each, have specificities toward different substrates and different lysosomal enzymes. It is clear that some patients with glucosylceramide storage have defects in saposin C (SAP-2). ${ }^{23,24}$ These patients have enlarged spleens and neurological disease similar to patients with juvenile Gaucher disease, but their glucocerebrosidase activity is normal. Some severely affected patients with HSM and neurological disease have been found to have mutations in the prosaposin gene resulting in none of the saposins being expressed. ${ }^{25}$

Another disorder caused by a defect in a genetically distinct sphingolipid activator protein is the AB variant of GM2 gangliosidosis. ${ }^{26}$ This protein, coded for on human chromosome 5, interacts with hexosaminidase A and GM2 ganglioside to facilitate the hydrolysis of GM2 ganglioside. ${ }^{6}$ This should be considered in any child with symptoms characteristic of TaySachs disease and Sandhoff disease and whose total $\beta$-hexosaminidase and hexosaminidase A are normal. These patients have an excess of GM2 ganglioside in cerebrospinal fluid. ${ }^{27}$ Molecular studies could identify the mutation(s) in the GM2 activator gene. Also, some patients with GM2 gangliosidosis have normal total $\beta$-hexosaminidase and percent hexosaminidase A when measured with synthetic $\beta$ - $N$-acetylglucosaminyl substrate and heat denaturation. These patients with the socalled B1 variant of GM2 gangliosidosis can be reliably diagnosed using the sulfated $\beta-N$-acetylglucosaminyl substrate. Thus the sulfated hexosaminidase substrate should be used when screening undiagnosed patients.

Four genetically distinct diseases result in either the lysosomal storage of bound sialic acid or free sialic acid or the accumulation and excretion of a large amount of free sialic acid. Galactosialidosis is caused by a defect in protective protein and 


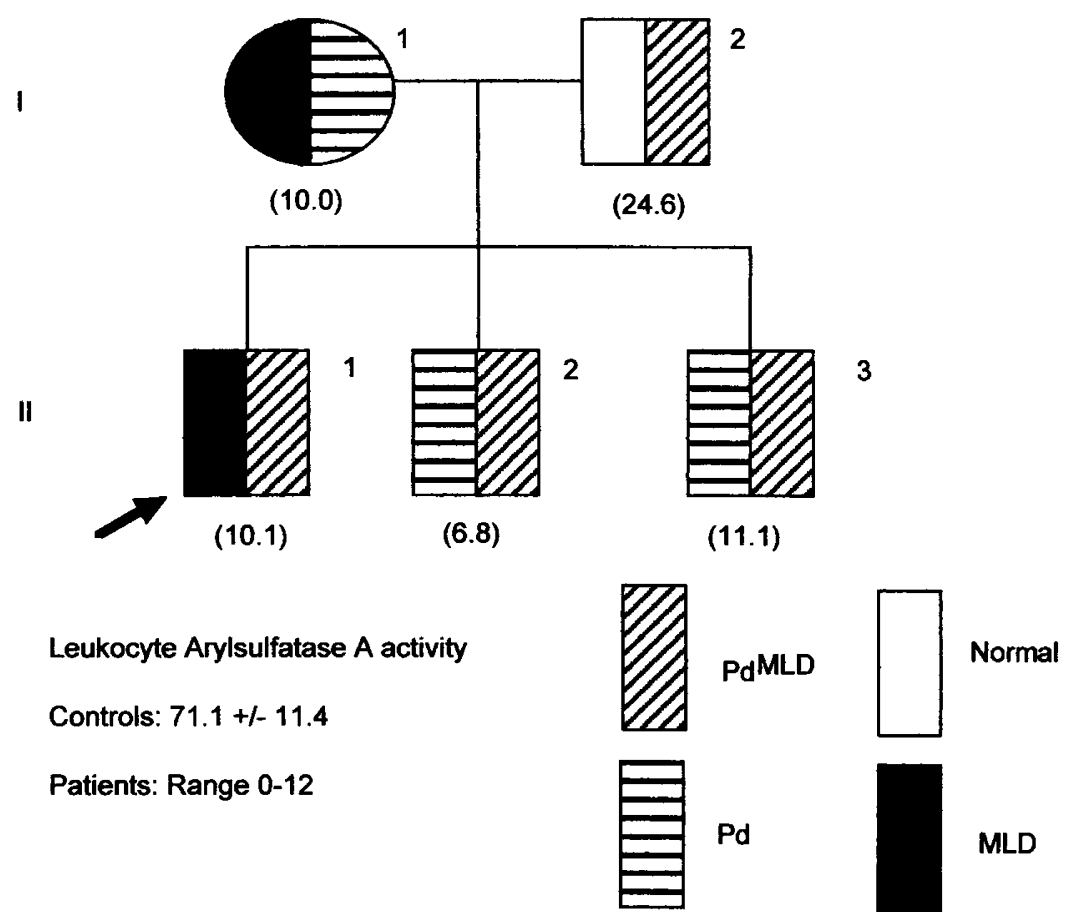

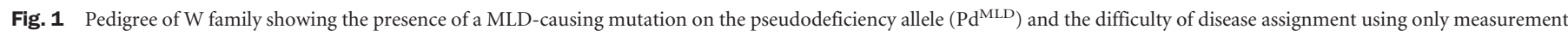

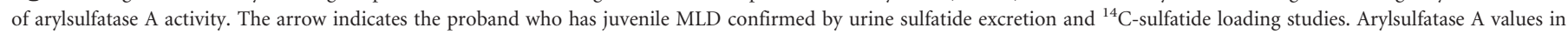
leukocytes in all family members and the four different alleles present in this family are illustrated.

was discussed above. In sialidosis, only sialidase activity is deficient. Patients can present as neonates with NIFH or nephrosis; as infants with HSM, coarse facial features, dysostosis multiplex, and developmental delay; or as older patients with myoclonus, cherry red spots, and ataxia. While sialidase cannot be measured in previously frozen leukocytes, a finding of increased bound sialic acid in leukocytes would suggest this diagnosis, and studies in cultured skin fibroblasts would offer a confirmation. Because sialic acid is easy to quantitate and the concentration range per milligram of protein is surprisingly tight, it is worth measuring in any patient with some of the key clinical features mentioned above. If the total sialic acid concentration is elevated as initially determined in an acid hydrolyzed sample, the sialic acid concentration can be measured in another aliquot without acid hydrolysis. If that is also elevated, then a disorder involving the accumulation of free sialic acid is the problem. Most patients with defects in the export of sialic acid from the lysosomes are severely affected. ${ }^{28}$ However, the patients from Finland with Salla disease who have a defect in the same gene tend to be mildly affected. A nonlysosomal disorder that will be detected in a screen of sialic acid content is sialuria. ${ }^{29}$ This is a relatively mild disorder that results from the excessive synthesis of sialic acid due to the lack of feedback inhibition at the UDP- $N$-acetyl-glucosamine-2-epimerase step. Recent studies have demonstrated that this disease could be inherited as a dominant trait. Patients with sialic acid storage disease and sialuria will both excrete excessive amounts of free sialic acid in urine.

\section{Prenatal testing for lysosomal storage diseases}

Many of the LSD are very severe, and they can result in early death without the availability of effective therapy. Most couples who are presented with a diagnosis of a LSD in their child will obtain genetic counseling before subsequent pregnancies. Although many are aware of the availability of prenatal diagnosis after conception, some families inquire about preimplantation diagnosis. ${ }^{30}$ While the disease-causing mutations can be identified in most patients, the successful use of this technology in more pregnancies must be demonstrated before its use can be encouraged. On the other hand, prenatal diagnosis is extremely accurate for most LSD using direct chorionic villus samples, cultured trophoblasts, and cultured amniotic fluid cells. Those disorders diagnosed by enzymatic assay usually can be analyzed within 1 day from a chorionic villus sample taken at 9.5 weeks' gestation. Very low values for the enzyme in question and normal values for other enzymes complete the diagnosis, and the couple can act on this information. Studies on cultured trophoblasts from the same original sampling should confirm the prediction of an affected or unaffected fetus. Of course, amniotic fluid cells have been used for the diagnosis of LSD for more than three decades. Knowing the mutation(s) in a proband does not necessarily make the prenatal testing more accurate or faster and is rarely used in this laboratory.

\section{Methods for early diagnosis}

There have been recent attempts to develop tests to screen newborn infants who have no family history of a LSD using samples obtained at birth. ${ }^{31-33}$ These tests would utilize either 
blood spots obtained from heal sticks to measure the activity of certain lysosomal enzymes or detect abnormal analytes or small plasma samples to determine the concentrations of certain proteins, including lysosome-associated membrane proteins (LAMP-1 and -2) and saposins. The utilization of dried blood spots for enzyme studies does seem feasible, but without a family history of one LSD, a large number of extra blood spots would be needed to cover even the most common LSD. Unless mutation analysis revealed a mutation(s) clearly associated with a phenotype, prediction of the clinical course in an untreated patient would be difficult. However, the identification of a patient at birth would result in careful clinical and laboratory observation, and treatment could begin at the earliest possible time.

The finding of elevated LAMP- 1 and -2 and certain saposins in plasma from newborns could indicate a diagnosis of a LSD. $^{31,32}$ While not all LSD show elevated levels of these proteins, some clearly do. Any patient with elevated levels would have to be studied in detail to determine which, if any, LSD was the cause of the elevation. With a positive test expected in about 1 in 10,000 births in the United States, a further battery of testing would be required in about 400 newborns to determine the precise diagnosis. Many of the samples used to demonstrate elevated LAMP- 1 and -2 and saposins in plasma came from older, previously diagnosed patients. It remains to be demonstrated that these proteins are elevated in newborns affected with one of these disorders. Also, it would be difficult to predict the clinical course and need for treatment in patients diagnosed without a family history. Molecular studies looking for known mutations might be helpful, but genotype-phenotype correlations have not always been useful for predicting clinical course in later-onset patients.

\section{Predicting the clinical course in untreated patients}

When a LSD is diagnosed in a child or adult, one of the key questions asked, besides the availability of treatment, is what clinical course lies ahead for the patient. This information would also be helpful in selecting those patients who would benefit most from defect-correcting therapy. Residual enzymatic activity, as measured in leukocytes, plasma, or cultured skin fibroblasts using natural or synthetic substrates, generally is not an accurate predictor of phenotype. A number of studies have tried to correlate phenotype by measuring both enzymatic activity and amount of antigen present in cultured skin fibroblasts. $^{34,35}$ This requires controlled growing conditions and monospecific antibodies which are not readily available. Since we rarely require cultured skin fibroblasts for diagnosis, we have not pursued this method.

Mutation analysis of all newly diagnosed patients would be useful for cataloging purposes and possibly for helping with carrier identification in relatives. While molecular analysis may identify mutations that can predict the presence or absence of neurological disease (e.g., N370S in glucocerebrosidase), it is less good at predicting the clinical course of an untreated patient. Careful evaluation of these patients may result in a more accurate prognosis. In those disorders with ju- venile and adult onset, as opposed to infantile onset, the prediction of clinical course is not very reliable. This happens to be especially true for some of the LSD in which ERT has become available. Gaucher disease, Fabry disease, and MPS I are three such LSD with ERT available or in the final stages of evaluation. ${ }^{36-38}$ Other than testing for some common mutations and Pd alleles in certain LSD, we usually do not perform mutation analysis in newly diagnosed patients.

\section{Options for treatment}

For many patients with a LSD, supportive care is the only option at this time (Table 1). However, an increasing number of patients with certain disorders are undergoing HSCT and ERT in an attempt to slow the course of the disease, prevent the onset of some clinical features, or reverse previous pathology. The first option has been considered in those patients for whom a suitable donor (HLA-matched or umbilical cord blood) is available and when the neurological symptoms are not severe. HSCT have been performed in more than $300 \mathrm{pa}-$ tients with a LSD. Some underwent transplantation more than two decades ago and have clearly had a positive response. ${ }^{39,40}$ Other HSCT were performed in only a few patients with a given disorder, providing no clear conclusion regarding effectiveness. In general, HSCT has been most effective in improving the visceral and hematological symptoms of the treated patients. Severe changes in the bones appear to be difficult to correct; however, with time and the transplantation of mesenchymal cells together with HSC, some improvements may be noted. The evidence from the treatment of Krabbe disease and MLD shows a clear positive effect if the patients are treated very early in life or while neurological symptoms are very mild. ${ }^{40}$ Because HSCT appears to provide donor-derived microglial cells in brain, it seems to have a positive effect on the central nervous system, helping the patient retain intellectual functioning. Defects in the peripheral nervous system appear to be more resistant to correction.

ERT started more that 15 years ago with the large-scale isolation of glucocerebrosidase from human placentas for the treatment of Gaucher disease. The treatment of the nonneurological forms of Gaucher disease with this original source of glucocerebrosidase and the later recombinant form has been very successful. ${ }^{41}$ Despite pressure from concerned families and health care providers, significant problems still remain before ERT can be tried for most LSD. The small number of patients with certain LSD makes the effort to produce and test the necessary enzyme unprofitable. Significant involvement of the central nervous system and the rapid course of many disorders also makes it unlikely that intravenously supplied enzyme would reach the brain in significant quantities to reverse damage that occurred early in life or possibly during fetal development. This uncertainty does not rule out the use of ERT for patients who have significant somatic involvement in addition to mental deficiencies. That these patients have fewer contractures, significant improvement in blood chemistry, and improved cardiac and kidney function remains a benefit of ERT. Correction of the skeletal changes seen in patients with 
certain MPS disorders may take more time and higher dosages of enzyme to achieve. For Gaucher disease and Fabry disease, the diagnosis of symptomatic individuals still provides a window for successful treatment even after many years of lipid accumulation.

Substrate deprivation as an adjunctive treatment for certain sphingolipidoses was proposed in the past by Norman Radin ${ }^{42}$ and is receiving renewed attention..$^{43,44}$ Because symptoms in the patients are thought to result from the accumulation of undegraded substrate, a slower rate of accumulation accomplished by increasing the level of the defective degradative enzyme or by slowing the rate of synthesis of substrate or both could prove to be effective. If the rate of substrate synthesis was slowed until it was approximately equal to the rate of degradation, little additional accumulation would occur. This theoretically could be accomplished by compounds such as L-cycloserine, which inhibits a very early step in the synthesis of sphingolipids, or $\mathrm{N}$-butyldeoxygalactonojirimycin, which inhibits the synthesis of glycosphingolipids derived from glucosylceramide. It must be noted that sphingolipids play very important functions in cellular metabolism including signal transduction, cell adhesiveness, and nerve impulse transmission, and modification of these and other functions by these drugs in a developing child must be carefully tested in animal models. Because patients with the milder forms of the sphingolipidoses are thought to have some residual enzymatic activity, these drugs may provide some benefit. The amount of enzyme needed in ERT for Gaucher disease or Fabry disease could possibly be lowered if an inhibitor of glucosylceramide synthesis was also provided. However, these drugs also have significant side effects that may limit their use.

What is the state of gene therapy for the treatment of LSD? No gene therapy protocols are in active use for the treatment of patients with LSD. Many papers have described successful in vivo and ex vivo therapy trials using a large number of viral vectors in the considerable number of available animal models with LSD. A majority of these studies have been done using the animal models of MPS VII. ${ }^{45-50}$ These naturally occurring models have been used for many treatment studies because of the ease of detecting the clearance of storage products from most organs examined and because a simple histochemical test for $\beta$-glucuronidase activity is available. Unfortunately, it may have been a poor choice to expend so much effort, as very few human patients with this disease survive long enough to ever be treated. While these studies may provide "proof of principle" results, it is difficult to see much ability to transfer the exact technology to other LSD. Differences in protein defects, enzyme processing and stability, storage products, disease time course, and organs affected make this assumption suspect. Developments in the viral transfer of genes into HSC for autologous HSCT would be very useful because allogeneic HSCT has proved successful for some of the LSD. The direct injection of recombinant viral vectors into brain usually shows only localized expression for relatively short periods of time..$^{51}$ It is clear that more research needs to be done before viral vectors can be used to treat patients with LSD.
In recent years the use of neural and embryonic stem cells to treat a variety of genetic disorders has been proposed, and some have been utilized in animal models. ${ }^{52-54}$ Injection of neural stem cells, whether transduced with the gene of interest or not, into the ventricles of a young patient could result in widespread distribution of donor cells. Many more studies to isolate stem-like cells from patients for transduction and reimplantation into specific tissues, especially brain, are needed. Eventually it may take more than one treatment procedure to "cure" a patient with certain LSD-one acting quickly to prevent storage and arrest degeneration and one providing longterm correction.

\section{Acknowledgments}

Supported in part by grant DK38795 from the National Institutes of Health. The authors thank the many health care providers for sending samples and clinical information on their patients, the technicians who have worked in the Lysosomal Diseases Testing Laboratory since its inception in 1973, and the families who continue to provide us with inspiration to try harder to diagnose and to treat these diseases.

\section{References}

1. Poorthuis BJHM, Wevers RA, Kleijer WJ, Groener JEM, de Jong JGN, Van Weely S, Niezen-Koning KE, Van Diggelen OP. The frequency of lysosomal storage diseases in the Netherlands. Hum Genet 1999;105:151-156.

2. Meikle PJ, Hopwood JJ, Clague AE, Carey WF. Prevalence of lysosomal storage disorders. JAMA 1999;281:249-254.

3. Nelson J. Incidence of the mucopolysaccharidoses in Northern Ireland. Hum Genet 1997;101:355-358.

4. Gieselmann V. Lysosomal storage diseases. Biochim Biophys Acta 1994;1270:103136.

5. Hopwood JJ, Morris CP. The mucopolysaccharidoses: diagnosis, molecular genetics and treatment. Mol Biol Med 1990;7:381-404.

6. Kolter T, Sandhoff K. Recent advances in the biochemistry of sphingolipidoses. Brain Pathol 1998;8:79-100.

7. Stone DL, Sidransky E. Hydrops fetalis: lysosomal storage disorders in extremis. $A d v$ Pediatr 1999;46:409-440.

8. Vanier MT, Wenger DA, Comly ME, Rousson R, Brady RO, Pentchev PG. Niemann-Pick Group C: clinical variability and diagnosis based on defective cholesterol esterification. Clin Genet 1988;33:331-348.

9. Wenger DA, Williams C. Screening for lysosomal disorders. In: Hommes FA, editor Techniques in diagnostic human biochemical genetics: A laboratory manual. New York: Wiley-Liss, 1991:587-617.

10. Wenger DA, Tarby TJ, Wharton C. Macular cherry-red spots and myoclonus with dementia: coexistent neuraminidase and $\beta$-galactosidase deficiencies. Biochem Biophys Res Commun 1978;82:589-595.

11. d'Azzo A, Hoogeveen A, Reuser AJ, Robinson D, Galjaard H. Molecular defect in combined beta-galactosidase and neuraminidase deficiency in man. Proc Natl Acad Sci US A 1982;79:4535-4539.

12. Dubois G, Turpin JC, Baumann N. Absence of ASA activity in healthy father of a patient with metachromatic leukodystrophy [letter]. N Engl J Med 1975;293:302.

13. Baldinger S, Pierpont ME, Wenger DA. Pseudodeficiency of arylsulfatase A: a counseling dilemma. Clin Genet 1987;31:70-76.

14. Gieselmann V, Polten A, Kreysing J, Von Figura K. Arylsulphatase A pseudodeficiency: loss of a polyadenylation signal and N-glycosylation site. Proc Natl Acad Sci U S A 1989;86:9436-9440.

15. Gieselmann V, Fluharty AL, Tonnesen T, Von Figura K. Mutations in the arylsulfatase A pseudodeficiency allele causing metachromatic leukodystrophy. Am J Hum Genet 1991;49:407-413.

16. Shapiro LJ, Aleck KA, Kaback MM, Itabashi H, Desnick RJ, Brand N, Stevens RL Fluharty AL, Kihara H. Metachromatic leukodystrophy without arylsulfatase A deficiency. Pediatr Res 1979;13:1179-1181.

17. Rafi MA, Zhang X-L, DeGala G, Wenger DA. Detection of a point mutation in sphingolipid activator protein-1 mRNA in patients with a variant form of metachromatic leukodystrophy. Biochem Biophys Res Commun 1990;166:1017-1023. 
18. Millat G, Marcais C, Tomasetto C, Chikh K, Fensom AH, Harzer K, Wenger DA, Ohno K, Vanier MT. Niemann-Pick C1 disease: correlations between NPC1 mutations, levels of NPC1 protein and phenotypes emphasize the functional significance of the putative sterol-sensing domain and of the cysteine-rich luminal loop. Am J Hum Genet 2001;68:1373-1385.

19. Millat G, Chikh K, Naureckiene S, Sleat DE, Fensom AH, Higaki K, Elleder M, Lobel P, Vanier MT. Niemann-Pick disease type C: spectrum of HE1 mutations and genotype/phenotype correlations in the NPC2 group. Am J Hum Genet 2001;69:10131021.

20. Roff CF, Goldin E, Comly ME, Blanchette-Mackie J, Cooney A, Brady RO, Pentchev PG. Niemann-Pick type C disease: deficient intracellular transport of exogenously derived cholesterol. Am J Med Genet 1992;42:593-598.

21. Millat G, Marcais C, Rafi MA, Yamamoto T, Morris JA, Pentchev PG, Ohno K, Wenger DA, Vanier MT. Niemann-Pick C1 disease: the I1061T substitution is a frequent mutant allele in patients of Western European descent and correlates with a classical juvenile phenotype. Am J Hum Genet 1999;65:1321-1329.

22. Rorman EG, Grabowski GA. Molecular cloning of human co-beta-glucosidase cDNA: evidence that four sphingolipid hydrolase activator proteins are encoded by single genes in humans and rats. Genomics 1989;5:486-492.

23. Christomanou H, Chabas A, Pampols T, Guardiola A. Activator protein deficient Gaucher's disease: a second patient with the newly identified lipid storage disorder. Klin Wochenschr 1989;67:999-1003.

24. Rafi MA, de Gala G, Zhang X-L, Wenger DA. Mutational analysis in a patient with a variant form of Gaucher disease caused by SAP-2 deficiency. Somat Cell Mol Genet 1993;19:1-7.

25. Bradova V, Smid F, Ulrich-Bott B, Roggendorf W, Paton BC, Harzer K. Prosaposin deficiency: further characterization of the sphingolipid activator protein-deficient sibs. Multiple glycolipid elevations (including lactosylceramidosis), partial enzyme deficiencies and ultrastructure of the skin in this generalized sphingolipid storage disease. Hum Genet 1993;92:143-152.

26. Conzelmann E, Sandhoff $\mathrm{K}$. AB variant of infantile $\mathrm{G}_{\mathrm{M} 2}$ gangliosidosis: deficiency of a factor necessary for stimulation of hexosaminidase A-catalyzed degradation of ganglioside $\mathrm{G}_{\mathrm{M} 2}$ and glycolipid $\mathrm{G}_{\mathrm{A} 2}$. Proc Natl Acad Sci U S A 1978;75:3979-3983.

27. Kotagal S, Wenger DA, Alcala $\mathrm{H}$, Gomez $\mathrm{C}$, Horenstein $\mathrm{S}$. AB variant $\mathrm{G}_{\mathrm{M} 2}$ gangliosidosis: cerebrospinal fluid and neuropathological characteristics. Neurology 1986; $36: 438-440$.

28. Lemyre E, Russo P, Melancon SB, Gagne R, Potier M, Lambert M. Clinical spectrum of infantile free sialic acid storage disease. Am J Med Genet 1999;82:385-391.

29. Leroy JG, Seppala R, Huizing M, Dacremont G, De Simpel H, Van Coster RN, Orvisky E, Krasnewich DM, Gahl WA. Dominant inheritance of sialuria, an inborn error of feedback inhibition. Am J Hum Genet 2001;68:1419-1427.

30. Handyside AH, Delhanty JDA. Preimplantation genetic diagnosis: strategies and surprises. Trends Genet 1997;13:270-275.

31. Hua CT, Hopwood JJ, Carlsson SR, Harris RJ, Meikle PJ. Evaluation of the lysosome-associated membrane protein LAMP-2 as a marker for lysosomal storage disorders. Clin Chem 1998;44:2094-2102.

32. Chang MHY, Bindloss CA, Grabowski GA, Qi X, Winchester B, Hopwood JJ, Meikle PJ. Saposins A, B, C, and D in plasma of patients with lysosomal storage disorders. Clin Chem 2000;46:167-174.

33. Chamoles NA, Blanco MB, Gaggioli D, Casentini C. Hurler-like phenotype: enzymatic diagnosis in dried blood spots on filter paper. Clin Chem 2001;47:2098-2102.

34. Ashton LJ, Brooks DA, McCourt PAG, Muller VJ, Clements PR, Hopwood JJ. Immunoquantification and enzyme kinetics of $\alpha$-L-iduronidase in cultured fibroblasts from normal controls and mucopolysaccharidosis type I patients. Am J Hum Genet 1992;50:787-794.

35. Perkins KJ, Muller V, Weber B, Hopwood JJ. Prediction of Sanfilippo phenotype severity from immunoquantification of heparan- $\mathrm{N}$-sulfamidase in cultured fibro- blasts from mucopolysaccharidosis type IIIA patients. Mol Genet Metab 2001;73: 306-312.

36. Barton NW, Brady RO, Dambrosia JM, Di Bisceglie AM, Doppelt SH, Hill SC, Mankin HJ, Murray GJ, Parker RI, Argoff CE. Replacement therapy for inherited enzyme deficiency: macrophage-targeted glucocerebrosidase for Gaucher's disease. N Engl J Med 1991;324:1464-1470.

37. Eng CM, Guffon N, Wilcox WR, Germain DP, Lee P, Waldek S, Caplan L, Linthorst GE, Desnick RJ. Safety and efficacy of recombinant human $\alpha$-galactosidase A replacement therapy in Fabry's disease. N Engl J Med 2001;345:9-16.

38. Kakkis ED, Muenzer J, Tiller GE, Waber L, Belmont J, Passage M, Izykowski B, Phillips J, Doroshow R, Walot I, Hoft R, Neufeld EF. Enzyme replacement therapy in mucopolysaccharidosis I. N Engl J Med 2001;344:182-188.

39. Vellodi A, Young EP, Cooper A, Wraith JE, Winchester B, Meaney C, Ramaswami U, Will A. Bone marrow transplantation for mucopolysaccharidosis type I: experience in two British centres. Arch Dis Child 1997;76:92-99.

40. Krivit W, Aubourg P, Shapiro E, Peters C. Bone marrow transplantation for globoid cell leukodystrophy, adrenoleukodystrophy, metachromatic leukodystrophy, and Hurler syndrome. Curr Opin Hematol 1999;6:377-382.

41. Grabowski GA, Leslie N, Wenstrup R. Enzyme therapy for Gaucher disease: the first 5 years. Blood Rev 1998;12:115-133.

42. Vunnam RR, Radin NS. Analogs of ceramide that inhibit glucocerebroside synthetase in mouse brain. Chem Phys Lipids 1980;26:265-278.

43. Sundaram KS, Lev M. Inhibition of cerebroside synthesis in the brains of mice treated with L-cycloserine. J Lipid Res 1985;26:473-477.

44. Andersson U, Butters TD, Dwek RA, Platt FM. N-Butyldeoxygalactonojirimycin: a more selective inhibitor of glycosphingolipid biosynthesis than $N$-butyldeoxynojirimycin, in vitro and in vivo. Biochem Pharmacol 2000;59:821-829.

45. Ghodsi A, Stein C, Derksen T, Yang G, Anderson RD, Davidson BL. Extensive $\beta$-glucuronidase activity in murine central nervous system after adenovirus-mediated gene transfer to brain. Hum Gene Ther 1998;9:2331-2340.

46. Elliger SS, Elliger CA, Aguilar CP, Raju NR, Watson GL. Elimination of lysosomal storage in brains of MPS VII mice treated by intrathecal administration of an adenoassociated virus vector. Gene Ther 1999;6:1175-1178.

47. Daly TM, Okuyama T, Vogler C, Haskins ME, Muzyczka N, Sands MS. Neonatal intramuscular injection with recombinant adeno-associated virus results in prolonged $\beta$-glucuronidase expression in situ and correction of liver pathology in mucopolysaccharidosis type VII mice. Hum Gene Ther 1999;10:85-94.

48. Bosch A, Perret E, Desmaris N, Trono D, Heard JM. Reversal of pathology in the entire brain of mucopolysaccharidosis type VII mice after lentivirus-mediated gene transfer. Hum Gene Ther 2000;11:1139-1150.

49. Serguera C, Sarkis C, Ridet JL, Colin P, Moullier P, Mallet J. Primary adult human astrocytes as an ex vivo vehicle for $\beta$-glucuronidase delivery in the brain. Mol Ther 2001;3:875-881.

50. Xu L, Haskins ME, Melniczek JR, Gao C, Weil MA, O’Malley TM, O’Donnell PA, Mazrier H, Ellinwood NM, Zweigle J, Wolfe JH, Ponder KP. Transduction of hepatocytes after neonatal delivery of a moloney murine leukemia virus based retroviral vector results in long-term expression of $\beta$-glucuronidase in mucopolysaccharidosis VII dogs. Mol Ther 2002;5:141-153.

51. Latchman DS. Herpes virus vectors for gene therapy in the nervous system. Biochem Soc Trans 1999;27:847-851.

52. Svendsen CN, Smith AG. New prospects for human stem-cell therapy in the nervous system. Trends Neurosci 1999;22:357-364.

53. Gage FH. Mammalian neural stem cells. Science 2000;287:1433-1438.

54. McKay R. Stem cells: hype and hope. Nature 2000;406:361-364. 